\title{
The association between driving a car and retention of brain volume in Japanese older adults
}

\author{
Hiroyuki Shimada ( $\nabla$ shimada@ncgg.go.jp ) \\ National Center for Geriatrics and Gerontology \\ Seongryu Bae \\ National Center for Geriatrics and Gerontology \\ Kenji Harada \\ National Center for Geriatrics and Gerontology \\ Keitaro Makino \\ National Center for Geriatrics and Gerontology \\ Ippei Chiba \\ National Center for Geriatrics and Gerontology

\section{Osamu Katayama} \\ National Center for Geriatrics and Gerontology

\section{Sangyoon Lee} \\ National Center for Geriatrics and Gerontology
}

\section{Research Article}

Keywords: structural brain volume, hippocampal brain atrophy, driving

Posted Date: June 4th, 2021

DOI: https://doi.org/10.21203/rs.3.rs-584908/v1

License: (c) (1) This work is licensed under a Creative Commons Attribution 4.0 International License.

Read Full License 


\title{
The association between driving a car and retention of brain volume in Japanese older adults
}

Hiroyuki Shimada, PhD a, ${ }^{*}$, Seongryu Bae, PhDa, Kenji Harada, MS a, Keitaro Makino, PhD a, Ippei Chiba, MS a, Osamu Katayama, PhD a, Sangyoon Lee, $\mathrm{PhD}$ a

a * Department of Preventive Gerontology, Center for Gerontology and Social Science, National Center for Geriatrics and Gerontology, 7-430 Morioka, Obu, Aichi 474-8511, Japan

*shimada@ncgg.go.jp

\author{
Abstract \\ Objective: To examine the association between driving and structural brain volume in older individuals.
}

Methods: In this cross-sectional study, high-resolution magnetic resonance imaging was performed in 1063 older adults. We examined global brain measures, including gray and white matter volumes and subcortical volume, using the FreeSurfer program. Participants were divided into non-drivers, those who drove $<7$ days a week, and every day drivers. They were further classified into a non-driving group, an active group (drove $10 \mathrm{~km}$ at least once a week), and a less-active group (drove $10 \mathrm{~km}$ less than once a week).

Results: Drivers had a larger hippocampal volume than non-drivers $(p=0.048)$. Low-frequency drivers had a larger occipital cortex volume than non-drivers and high-frequency drivers $(p=0.007)$. Active drivers had larger temporal cortex volumes than non-active drivers $(p=0.020)$, larger cingulate cortex volumes than non-drivers and less-active drivers $(p=0.002)$, and larger hippocampus volumes than non-drivers $(p=0.019)$. A post-hoc analysis revealed no significant between-group differences in the amygdala.

Conclusions: Driving was associated with diminished hippocampal brain atrophy in older adults. Active drivers with a larger life space exhibited less brain atrophy in several regions, including the temporal and cingulate cortices.

\section{Introduction}

Driving cessation is a major negative life event and is associated with a decline in general health, ${ }^{1}$ depressive symptoms, ${ }^{2}$ cognitive decline, ${ }^{3}$ increased risk for long-term care institutionalization, ${ }^{4}$ and increased mortality. ${ }^{5}$ Driving cessation also has a significant impact on the cost of health care. ${ }^{6}$ From a cognitive perspective, previous cohort studies have reported that driving a car is associated with the incidence of dementia in older adults ${ }^{7}$ and reversion from mild cognitive impairment $(\mathrm{MCl})$ to normal cognition. ${ }^{8}$ These studies suggest that driving a car is beneficial for health maintenance, including brain health, in older populations. One explanation for the positive relationship between driving and health is the increase in activity due to the expansion of life space. Similarly, previous studies have identified an association between constricted life space and an increased risk of frailty, ${ }^{9}$ mortality, ${ }^{9}$ Alzheimer's disease, and $\mathrm{MCl} .{ }^{10}$ Although it is clear that physical activity is an effective intervention for preventing frailty among older adults, ${ }^{11}$ the factors contributing to the association of constricted life space with an increased risk of dementia remain unknown.

In the present study, we examined the association between driving, life space, and brain volume in older participants in the National Center for Geriatrics and Gerontology-Study of Geriatric Syndromes (NCGG-SGS) in Japan.

Methods

\section{Standard protocol approvals, registrations, and patient consent}

The National Center for Geriatrics and Gerontology Institutional Review Board reviewed and approved this project (approval number: 1440-2). All individuals provided written informed consent prior to participation. This study was 
developed in accordance with the Declaration of Helsinki.

\section{Study design}

This was a cross-sectional study that examined the association between driving and differences in structural brain measurements.

\section{Participants}

Participants were recruited from a sub-cohort of the NCGG-SGS. ${ }^{12}$ This sub-cohort targeted adults aged $\geq 60$ years who lived in Takahama City, Aichi Prefecture, Japan. A total of 4,122 individuals participated in the survey, which included physical examinations, cognitive tests, and questionnaires. Invitation letters for brain magnetic resonance imaging (MRI) assessment were sent to 4,080 of the participants, excluding those who had a pacemaker. In total, 1,220 older adults participated in the MRI assessment. Participants were excluded because of missing data $(n=5)$, driving cessation $(n=39)$, MRI contraindications $(n=20)$, a history of stroke $(n=45)$, Parkinson's disease $(n=2)$, depression $(n=30)$, dementia $(n=1)$, and other brain diseases, including brain tumors and chronic subdural hematomas $(n=15)$. Following exclusion, data from the remaining 1,063 participants were analyzed.

\section{Driving status and life space assessment}

We identified drivers and non-drivers according to their yes/no responses to the item "Do you drive a motor vehicle?". ${ }^{13}$ In addition, drivers were asked how frequently they drove each week. To assess life space, the participants were asked about the frequency at whether they drove more than $10 \mathrm{~km}$ in a week. Participants were divided into three groups based on driving frequency, including a non-driving group, a low-frequency group (those who drove less than 7 days per week), and a high-frequency group (those who drove every day). Participants were also classified into the three following groups based on their life space: a non-driving group, an active driving group (those who drove $10 \mathrm{~km}$ at least once per week), and a less-active driving group (those who drove $10 \mathrm{~km}$ less than once a week).

\section{MRI acquisition}

Structural MRI was performed using a Siemens MAGNETOM Trio Tim 3T scanner (Siemens Medical Solutions, Erlangen, Germany) with a 12-channel head coil. A whole-brain three-dimensional T1-weighted magnetization prepared rapid acquisition gradient echo sequence was acquired in the sagittal plane with the following parameters: repetition time $=1800 \mathrm{~ms}$, echo time $=1.99 \mathrm{~ms}$, flip angle $=9^{\circ}$, slices $=160$, slice thickness $=1.1 \mathrm{~mm}$, voxel $=1.0 \times 1.0 \times 1.1 \mathrm{~mm}$, image matrix $=256 \times 256 \mathrm{~mm}$, and field of view $=250 \mathrm{~mm}$. Each scan took 4:06 $\mathrm{min}$ to complete. ${ }^{14}$

\section{Cortical and subcortical volume measurements}

Cortical and subcortical volumes were estimated using FreeSurfer version 6.0 (running in Linux Ubuntu 16.04 LTS). Each image was processed by running the standard "recon-all" pipeline. Details of the technical procedures have been published previously ${ }^{15}$ and are freely available for download (http://surfer.nmr.mgh.harvard.edu/). Briefly, analytical procedures included the removal of nonbrain tissue, ${ }^{16}$ automated Talairach transformation, volumetric segmentation of the subcortical white matter (WM) and deep gray matter (GM), ${ }^{17}$ intensity normalization, ${ }^{18}$ tessellation of the GM-WM boundary, automated topology correction, ${ }^{19}$ and accurate surface deformation to identify tissue borders. ${ }^{20}$ The FreeSurfer output underwent visual quality control and manual correction whenever necessary, and the steps were repeated for accuracy.

Cortical volume parcellation and region of interest (ROI) definition were performed using the DesikanKilliany atlas. ${ }^{20}$ Subcortical segmentation was based upon an existing atlas containing probabilistic information on the location of structures..$^{21}$ Global brain measures, such as total brain volume, total GM volume, total WM volume, and the sum of the cortical volume of all 34 ROIs, were analyzed. Individual Desikan-Killiany ROIs were combined into five regions (sum of the left and right hemispheres) for regional cortical volume analysis, including the frontal (superior frontal; rostral and caudal middle frontal; pars opercularis, pars triangularis, and pars orbitalis; lateral and medial orbitofrontal; precentral; paracentral; and frontal pole), parietal (superior parietal, inferior parietal, supramarginal, postcentral, and precuneus), temporal (superior, middle, and inferior temporal; banks of the superior temporal sulcus; fusiform; transverse temporal; entorhinal; temporal pole; and parahippocampal), occipital 
(lateral occipital, lingual, cuneus, and pericalcarine), and cingulate (rostral anterior, caudal anterior, posterior, and isthmus) cortices. In addition, five deep brain regions were analyzed, including the hippocampus, caudate, putamen, thalamus, and amygdala. ${ }^{22}$

\section{Potential confounding factors}

Based on previous studies, the influence of age, ${ }^{23}$ sex, ${ }^{24}$ educational attainment, ${ }^{25}$ hypertension, ${ }^{26}$ diabetes mellitus, ${ }^{27}$ hyperlipidemia, ${ }^{28}$ and cigarette smoking ${ }^{28}$ were controlled for in the analysis to adjust for the effects of factors that accelerate brain atrophy. To adjust for differences in head size across participants, regression modeling was performed with estimated total intracranial volume as the independent variable and brain volume as the outcome variable, and the regression residuals were used for subsequent analyses.

\section{Statistical analyses}

Baseline characteristics were compared between the driver and non-driver groups using the t-test and Chi-square test. Analysis of variance and Chi-square test with adjusted standardized residuals were used to compare the baseline variables among the three groups based on driving frequency and life space. Residuals followed the $t$ distribution, with $t>1.96$ taken to indicate $p<0.05$ and $t>2.56$ taken to indicate $p<0.01$. Analysis of covariance was used to determine inter-group differences in cortical volume. Bonferroni post-hoc analyses were used to compare the cortical volumes between the groups. Potential correlative covariates were included in the multivariate model. An alpha value of 0.05 was used to determine statistical significance, and all data management and statistical computations were performed using SPSS 25.0 software (IBM Corp., Armonk, NY, USA).

\section{Results}

\section{Characteristics of the study population}

Non-drivers were older, less educated, and had less hypertension, and less hyperlipidemia, and included less smokers, a higher proportion of females, and had a lower brain volume in all regions than drivers (Table 1).

\section{Comparison of brain volume after adjusting for confounding factors}

Drivers had a significantly larger hippocampal volume than non-drivers $(F=3.914, p=0.048$; Table 2, Figure 1 , Figure 2). Group differences based on the driving frequency were found in the occipital cortex ( $F=5.044, p=0.007$; Table 2, Figure 2). Post-hoc analysis revealed that low-frequency drivers had a larger occipital cortex volume than non-drivers and high-frequency drivers. No significant difference was found between non-drivers and high-frequency drivers. Group differences were found in the temporal region $(F=3.922, p=0.020)$, cingulate cortices $(F=6.213, p=0.002)$, hippocampus $(F=3.968, p=0.019)$, and amygdala $(F=3.259, p=0.039)$ based on driving activity (Table 2, Figure 2 ). Post-hoc analysis revealed that active drivers had a larger temporal cortex volume than non-active drivers, a larger cingulate cortex volume than non-drivers and non-active drivers, and a larger hippocampus volume than non-drivers. No significant differences were observed in the amygdala by post-hoc analyses.

\section{Discussion}

Driving is a complex process that involves visuospatial recognition, planning, and execution of appropriate actions based on the traffic environment. ${ }^{29}$ Therefore, maintaining cognitive functions, such as visuospatial abilities and executive function, is key to ensuring the safety of older adults who drive. Previous studies have demonstrated that older drivers with a history of car accidents performed poorly on cognitive tasks requiring visuospatial abilities and executive function than an age-matched accident-free group. ${ }^{30}$ Evidence has also suggested that driving activates the brain with large variation and affects brain plasticity. ${ }^{31}$

The present study found that older individuals who drove more frequently maintained a larger brain volume in the hippocampus than non-drivers. A study on London taxi drivers reported that those who memorized and drove London's complex streets had greater GM volume in the posterior hippocampus than non-taxi drivers, and that the volume was positively correlated with the amount of time spent as a taxi driver. ${ }^{32,33}$ These findings suggest that the healthy adult brain undergoes local plastic structural changes in response to environmental stimuli. The hippocampus facilitates spatial navigational memory, and functional neuroimaging studies of navigation in humans has revealed 
that the retrieval of previously learned navigational information was associated with the activation of the posterior hippocampus. ${ }^{34}$ The results of the present study suggest that years of driving experience may routinely activate the hippocampus and contribute to the retention of hippocampal volume.

Comparing groups based on driving frequency revealed a significant difference in the occipital volume. The occipital lobe is divided into several functional visual cortices. The primary visual cortex provides low-level details of local orientation, spatial-frequency, and color properties within small receptive fields, which are all necessary for safe driving. ${ }^{35}$ Therefore, it is unsurprising that the occipital volume was higher in frequent drivers than in non-drivers; however, the post-hoc analysis revealed no relationship between driving frequency and occipital volume, which indicates a need for further studies. This result might be attributable to the lack of a quantitative evaluation of driving frequency. We plan to use a quantitative monitoring system, such as a driving recorder, in our future research.

Several reviews have suggested that physical activity has significant effects on brain volume, including the prefrontal cortex and hippocampus, but it remains unclear how effective physical activity is in preserving brain volume. ${ }^{36,37}$ Many studies have suggested that physical activity may be involved in the reduction of beta-amyloid accumulation and in increasing cognitive function, as well as of neuroinflammation factors such as tumor necrosis factor alpha, interleukin-1-beta, and interleukin-6, high-density lipoprotein levels, cerebral blood flow, and brainderived neurotrophic factor, and that these factors may contribute to brain health during aging. ${ }^{38}$ Physical inactivity has become endemic in industrialized countries, particularly among certain occupations, such as professional drivers. ${ }^{39}$ Inactivity is attributed to the fact that the overall risk for coronary heart disease in particular (and possibly other conditions) is higher than any other lifestyle risk factor. ${ }^{40}$ However, cars are often a necessity for older people living in the community if they wish to go out, and driving cessation significantly increases the need for care in daily life. ${ }^{12} \mathrm{~A}$ previous study found that older drivers were more physically active (i.e., demonstrated less sedentary time), potentially because of increased access to outdoor activities or a larger social network. ${ }^{41}$ The results of the present study suggest that maintaining an active lifestyle with the use of a car was beneficial for the retention of brain volume in the temporal lobe, hippocampus, cingulate cortex, and amygdala in older adults.

Visual information from the visual cortex is processed in the temporal lobe via the ventral stream, which plays an important role in visual object identification. ${ }^{42}$ Therefore, it is possible that the volume of the temporal lobe was maintained in older adults who drove because they were more often engaged in identifying visual objects, such as other vehicles and road signs. ${ }^{42}$ The cingulate cortex contains many important neural circuits, including the center of the reward system, amygdala, lateral prefrontal cortex, parietal cortex, motor areas, spinal cord, hippocampus, and limbic regions. ${ }^{43}$ The dorsal anterior cingulate cortex projects directly to the spinal cord and the motor and limbic cortices. Several functions have been ascribed to this area, including attention-for-action/target selection, ${ }^{44}$ motor response selection, ${ }^{45}$ error detection/performance monitoring, ${ }^{46}$ and anticipation, ${ }^{47}$ which are all required for safe driving. The amygdala is a subcortical nucleus that has a variety of functions, including emotion recognition, social cognition, and reward learning. ${ }^{48,49}$ Currently, a direct functional relationship between the amygdala and driving is unclear.

The present study classified participants in two ways (i.e., according to driving frequency and driving distance); the differences in brain volume were more robust in regions when comparing distance rather than frequency. These findings may suggest that the beneficial effect of driving on brain volume depends on expansion of life space using a vehicle rather than driving frequency. Indeed, previous studies have reported that a constricted life space is associated with increased risk of Alzheimer's disease, $\mathrm{MCl}$, and cognitive decline among older persons..$^{10,50}$ Therefore, it may be important to develop effective strategies to support the continuation of safe driving and provide alternative means of transportation that allows older adults to maintain their daily life space.

The current study has several limitations. The cross-sectional design prevents exclusion of the possibility of reverse causality (i.e., changes in brain structure cause behavioral changes, including alterations in driving habits). Longitudinal studies are needed to examine whether driving status and activity are associated with diminished brain atrophy over time. Additionally, the possibility of residual confounding factors, such as an apolipoprotein E genotype, cannot be excluded. ${ }^{51}$

\section{Conclusions}

To our knowledge, this is the first study to demonstrate the relationship between driving and brain structure in older individuals. Given that brain atrophy is defined by multiple factors, an analysis with a large population is necessary to ensure statistical power to attribute brain atrophy to single factors. This study indicates that driving is associated with maintaining brain volume in several cortical areas in older individuals after adjusting for several confounding factors. 


\section{Data availability}

Additional anonymized data not available within the article or supplementary material are available to qualified researchers on reasonable request.

\section{References}

1 Edwards, J. D., Lunsman, M., Perkins, M., Rebok, G. W. \& Roth, D. L. Driving cessation and health trajectories in older adults. J Gerontol A Biol Sci Med Sci 64, 1290-1295, doi:10.1093/gerona/glp114 (2009).

2 Marottoli, R. A. et al. Driving cessation and increased depressive symptoms: prospective evidence from the New Haven EPESE. Established Populations for Epidemiologic Studies of the Elderly. J Am Geriatr Soc 45, 202-206 (1997). Choi, M., Lohman, M. C. \& Mezuk, B. Trajectories of cognitive decline by driving mobility: evidence from the Health and Retirement Study. Int J Geriatr Psychiatry 29, 447-453, doi:10.1002/gps.4024 (2013). Freeman, E. E., Gange, S. J., Munoz, B. \& West, S. K. Driving status and risk of entry into long-term care in older adults. Am J Public Health 96, 1254-1259, doi:10.2105/AJPH.2005.069146 (2006). Edwards, J. D., Perkins, M., Ross, L. A. \& Reynolds, S. L. Driving status and three-year mortality among community-dwelling older adults. J Gerontol A Biol Sci Med Sci 64, 300-305, doi:10.1093/gerona/gIn019 (2009). Guralnik, J. M., Alecxih, L., Branch, L. G. \& Wiener, J. M. Medical and long-term care costs when older persons become more dependent. Am J Public Health 92, 1244-1245 (2002).

7 Shimada, H., Makizako, H., Lee, S., Doi, T. \& Lee, S. Lifestyle activities and the risk of dementia in older Japanese adults. Geriatr Gerontol Int 18, 1491-1496, doi:10.1111/ggi.13504 (2018).

8 Shimada, H., Doi, T., Lee, S. \& Makizako, H. Reversible predictors of reversion from mild cognitive impairment to normal cognition: a 4-year longitudinal study. Alzheimers Res Ther 11, 24, doi:10.1186/s13195-019-0480-5 (2019).

9 Xue, Q. L., Fried, L. P., Glass, T. A., Laffan, A. \& Chaves, P. H. Life-space constriction, development of frailty, and the competing risk of mortality: the Women's Health And Aging Study I. Am J Epidemiol 167, 240-248, doi:kwm270 [pii]10.1093/aje/kwm270 (2008).

10 James, B. D., Boyle, P. A., Buchman, A. S., Barnes, L. L. \& Bennett, D. A. Life space and risk of Alzheimer disease, mild cognitive impairment, and cognitive decline in old age. Am J Geriatr Psychiatry 19, 961-969, doi:10.1097/JGP.0b013e318211c219 (2011).

11 Oliveira, J. S. et al. Evidence on Physical Activity and the Prevention of Frailty and Sarcopenia Among Older People: A Systematic Review to Inform the World Health Organization Physical Activity Guidelines. J Phys Act Health, 1-12, doi:10.1123/jpah.2020-0323 (2020).

12 Shimada, H. et al. Cognitive Impairment and Disability in Older Japanese Adults. PLoS One 11, e0158720, doi:10.1371/journal.pone.0158720 (2016). Anstey, K. J., Windsor, T. D., Luszcz, M. A. \& Andrews, G. R. Predicting driving cessation over 5 years in older adults: psychological well-being and cognitive competence are stronger predictors than physical health. J Am Geriatr Soc 54, 121-126, doi:10.1111/j.1532-5415.2005.00471.x (2006). Bae, S. et al. Engagement in Lifestyle Activities is Associated with Increased Alzheimer's Disease-Associated Cortical Thickness and Cognitive Performance in Older Adults. J Clin Med 9, doi:10.3390/jcm9051424 (2020).

15 Fischl, B. FreeSurfer. Neuroimage 62, 774-781, doi:10.1016/j.neuroimage.2012.01.021 (2012). Segonne, F. et al. A hybrid approach to the skull stripping problem in MRI. Neuroimage 22, 1060-1075, doi:10.1016/j.neuroimage.2004.03.032 (2004). Fischl, B. et al. Sequence-independent segmentation of magnetic resonance images. Neuroimage $\mathbf{2 3}$ Suppl 1, S69-84, doi:10.1016/j.neuroimage.2004.07.016 (2004).

18 Sled, J. G., Zijdenbos, A. P. \& Evans, A. C. A nonparametric method for automatic correction of intensity nonuniformity in MRI data. IEEE Trans Med Imaging 17, 87-97, doi:10.1109/42.668698 (1998). Segonne, F., Pacheco, J. \& Fischl, B. Geometrically accurate topology-correction of cortical surfaces using nonseparating loops. IEEE Trans Med Imaging 26, 518-529, doi:10.1109/TMI.2006.887364 (2007). Fischl, B. \& Dale, A. M. Measuring the thickness of the human cerebral cortex from magnetic resonance images. Proc Natl Acad Sci U S A 97, 11050-11055, doi:10.1073/pnas.200033797 (2000). Fischl, B. et al. Whole brain segmentation: automated labeling of neuroanatomical structures in the human brain. Neuron 33, 341-355, doi:10.1016/s0896-6273(02)00569-x (2002). Gu, Y. et al. Mediterranean diet and brain structure in a multiethnic elderly cohort. Neurology 85, 1744- 
1751, doi:10.1212/WNL.0000000000002121 (2015).

Good, C. D. et al. A voxel-based morphometric study of ageing in 465 normal adult human brains. Neuroimage 14, 21-36, doi:S1053-8119(01)90786-4 [pii]10.1006/nimg.2001.0786 (2001). Aging 32, 907-915, doi:10.1016/j.neurobiolaging.2009.05.003 (2011). Foubert-Samier, A. et al. Education, occupation, leisure activities, and brain reserve: a population-based study. Neurobiol Aging 33, 423 e415-425, doi:10.1016/j.neurobiolaging.2010.09.023 (2012). Strassburger, T. L. et al. Interactive effects of age and hypertension on volumes of brain structures. Stroke 28, 1410-1417, doi:10.1161/01.str.28.7.1410 (1997). Coffey, C. E. et al. Quantitative cerebral anatomy of the aging human brain: a cross-sectional study using magnetic resonance imaging. Neurology 42, 527-536, doi:10.1212/wnl.42.3.527 (1992). Meyer, J. S. et al. Risk factors accelerating cerebral degenerative changes, cognitive decline and dementia. Int J Geriatr Psychiatry 14, 1050-1061, doi:10.1002/(sici)1099-1166(199912)14:12<1050::aidgps56>3.0.co;2-z (1999).

Dewar, R., Olson, P. \& Alexanc Publishing Company, 2007). Owsley, C. et al. Visual processing impairment and risk of motor vehicle crash among older adults. JAMA 279, 1083-1088 (1998). Spiers, H. J. \& Maguire, E. A. Neural substrates of driving behaviour. Neuroimage 36, 245-255, doi:10.1016/j.neuroimage.2007.02.032 (2007). Maguire, E. A. et al. Navigation-related structural change in the hippocampi of taxi drivers. Proc Natl Acad Sci U S A 97, 4398-4403, doi:10.1073/pnas.070039597 (2000). Maguire, E. A., Woollett, K. \& Spiers, H. J. London taxi drivers and bus drivers: a structural MRI and neuropsychological analysis. Hippocampus 16, 1091-1101, doi:10.1002/hipo.20233 (2006). Maguire, E. A. et al. Knowing where and getting there: a human navigation network. Science 280, 921-924, doi:10.1126/science.280.5365.921 (1998). Xing, D., Ringach, D. L., Shapley, R. \& Hawken, M. J. Correlation of local and global orientation and spatial frequency tuning in macaque V1. J Physio/ 557, 923-933, doi:10.1113/jphysiol.2004.062026 (2004). Erickson, K. I. \& Kramer, A. F. Aerobic exercise effects on cognitive and neural plasticity in older adults. Br J Sports Med 43, 22-24, doi:10.1136/bjsm.2008.052498 (2009).

37 Hillman, C. H., Erickson, K. I. \& Kramer, A. F. Be smart, exercise your heart: exercise effects on brain and cognition. Nat Rev Neurosci 9, 58-65, doi:10.1038/nrn2298 (2008). Tokgoz, S. \& Claassen, J. Exercise as Potential Therapeutic Target to Modulate Alzheimer's Disease Pathology in APOE epsilon4 Carriers: A Systematic Review. Cardiol Ther, doi:10.1007/s40119-020-00209-z (2021). Korelitz, J. J. et al. Health habits and risk factors among truck drivers visiting a health booth during a trucker trade show. Am J Health Promot 8, 117-123, doi:10.4278/0890-1171-8.2.117 (1993). Taylor, A. H. \& Dorn, L. Stress, fatigue, health, and risk of road traffic accidents among professional drivers: the contribution of physical inactivity. Annu Rev Public Health 27, 371-391, doi:10.1146/annurev.publhealth.27.021405.102117 (2006). Amagasa, S. et al. Drivers Are More Physically Active Than Non-Drivers in Older Adults. International journal of environmental research and public health 15, doi:10.3390/ijerph15061094 (2018). Kohler, S., Kapur, S., Moscovitch, M., Winocur, G. \& Houle, S. Dissociation of pathways for object and spatial vision: a PET study in humans. Neuroreport 6, 1865-1868, doi:10.1097/00001756-19951002000011 (1995).

43 Jumah, F. \& Dossani, R. Neuroanatomy, Cingulate Cortex. StatPearls Publishing (2021). Posner, M. I., Petersen, S. E., Fox, P. T. \& Raichle, M. E. Localization of cognitive operations in the human brain. Science 240, 1627-1631, doi:10.1126/science.3289116 (1988). 924, doi:10.1038/13224 (1999). Gehring, W. J. \& Knight, R. T. Prefrontal-cingulate interactions in action monitoring. Nat Neurosci 3, 516520, doi:10.1038/74899 (2000).

47 Murtha, S., Chertkow, H., Beauregard, M., Dixon, R. \& Evans, A. Anticipation causes increased blood flow to the anterior cingulate cortex. Hum Brain Mapp 4, 103-112, doi:10.1002/(SICI)10970193(1996)4:2<103::AID-HBM2>3.0.CO;2-7 (1996). Janak, P. H. \& Tye, K. M. From circuits to behaviour in the amygdala. Nature 517, 284-292, doi:10.1038/nature14188 (2015).

49 Adolphs, R. What does the amygdala contribute to social cognition? Ann N Y Acad Sci 1191, 42-61, doi:10.1111/j.1749-6632.2010.05445.x (2010). 
50 Crowe, M. et al. Life-space and cognitive decline in a community-based sample of African American and Caucasian older adults. J Gerontol A Biol Sci Med Sci 63, 1241-1245, doi:63/11/1241 [pii] (2008).

51 Okonkwo, O. C. et al. Cerebral atrophy, apolipoprotein E varepsilon4, and rate of decline in everyday function among patients with amnestic mild cognitive impairment. Alzheimers Dement 6, 404-411, doi:10.1016/j.jalz.2010.02.003 (2010).

Funding

This research was supported by the AMED (grant numbers 15dk0107003h0003, 15dk0207004h0203) and JSPS (Grantin-Aid for Scientific Research (A), 26242059).

\section{Author information}

Affiliations

Centre for Gerontology and Social Science, National Centre for Geriatrics and Gerontology, 7-430, Morioka-cho, Obu City, Aichi Prefecture 474-8511, Japan

Hiroyuki Shimada, Seongryu Bae, Kenji Harada, Keitaro Makino, Ippei Chiba, Osamu Katayama, Sangyoon Lee

\section{Contributions}

All authors whose names appear on the submission 1) made substantial contributions to the conception or design of the work; or the acquisition, or interpretation of data; 2) revised it critically for important intellectual content; 3 ) approved the version to be published; and 4) agree to be accountable for all aspects of the work in ensuring that questions related to the accuracy or integrity of any part of the work are appropriately investigated and resolved. The first draft of the manuscript was written by H.S. and all the authors commented on previous versions of the manuscript. S.B. and K.H. made final dataset and figures.

\section{Corresponding author}

Correspondence to Hiroyuki Shimada.

\section{Ethics declarations}

Competing interests

The authors declare no competing interests. 


\begin{tabular}{|c|c|c|c|c|}
\hline & Total & Non-drivers & Drivers & $\mathrm{p}$ \\
\hline \multicolumn{5}{|c|}{ Demographics and confounding factors } \\
\hline Age, years: mean (SD) & $70.1(6.4)$ & $75.7(6.3)$ & $69.4(6)$ & $<0.001$ \\
\hline Education, years: mean (SD) & $11.8(2.5)$ & $10.1(2)$ & $12(2.4)$ & $<0.001$ \\
\hline Female, n (\%) & $515(48)$ & $107(92)$ & $408(43)$ & $<0.001$ \\
\hline Hypertension, n (\%) & $594(56)$ & $50(43)$ & $544(57)$ & 0.003 \\
\hline Diabetes, n (\%) & $928(87)$ & $99(85)$ & $829(88)$ & 0.50 \\
\hline Hyperlipidemia, n (\%) & $756(71)$ & $67(58)$ & $689(73)$ & 0.001 \\
\hline Smoking, n (\%) & & & & $<0.001$ \\
\hline Never smoked & $605(57)$ & $107(92)$ & $498(53)$ & \\
\hline Former smoker & $331(31)$ & $5(4)$ & $326(34)$ & \\
\hline Current smoker & $127(12)$ & $4(3)$ & $123(13)$ & \\
\hline \multicolumn{5}{|c|}{ Brain structural and volume measures, $\mathrm{mL}$ : mean (SD) } \\
\hline $\begin{array}{l}\text { Estimated total intracranial } \\
\text { volume }\end{array}$ & $1388.6(146.5)$ & $1284.4(138.0)$ & $1401.3(142.5)$ & $<0.001$ \\
\hline TGMV & $573.8(49.0)$ & $533.7(44.8)$ & $578.7(47.3)$ & $<0.001$ \\
\hline TWMV & $424.2(49.1)$ & $386.7(43.5)$ & $428.8(47.8)$ & $<0.001$ \\
\hline SCGMV & $51.4(4.8)$ & $47.8(4.4)$ & $51.8(4.6)$ & $<0.001$ \\
\hline SCV & $422.2(38.2)$ & $391.1(35.2)$ & $426.0(36.7)$ & $<0.001$ \\
\hline Frontal & $149.5(14.1)$ & $139.5(12.8)$ & $150.7(13.7)$ & $<0.001$ \\
\hline Temporal & $97.9(9.8)$ & $90.0(8.9)$ & $98.8(9.4)$ & $<0.001$ \\
\hline Parietal & $99.7(9.9)$ & $92.9(10.2)$ & $100.6(9.6)$ & $<0.001$ \\
\hline Occipital & $44.4(5.0)$ & $40.4(4.0)$ & $44.8(4.9)$ & $<0.001$ \\
\hline Cingulate & $17.6(2.1)$ & $16.1(2.0)$ & $17.8(2.1)$ & $<0.001$ \\
\hline Hippocampus & $7.7(0.8)$ & $7.0(0.8)$ & $7.8(0.8)$ & $<0.001$ \\
\hline Caudate & $6.2(0.9)$ & $5.9(1.0)$ & $6.3(0.9)$ & $<0.001$ \\
\hline Putamen & $8.5(1.2)$ & $8.0(1.3)$ & $8.6(1.1)$ & $<0.001$ \\
\hline Thalamus & $12.4(1.3)$ & $11.5(1.1)$ & $12.5(1.3)$ & $<0.001$ \\
\hline Amygdala & $3.1(0.4)$ & $2.7(0.4)$ & $3.1(0.4)$ & $<0.001$ \\
\hline
\end{tabular}

TGMV = total gray matter volume; TWMV = total white matter volume; SCGMV = subcortical gray matter volume; SCV = sum cortical volume 
Table 2. Cross-sectional association between driving status and brain measures

\begin{tabular}{|c|c|c|c|c|c|c|c|c|}
\hline & \multicolumn{2}{|c|}{ Driving status } & \multicolumn{3}{|c|}{ Driving frequency } & \multicolumn{3}{|c|}{ Life space } \\
\hline & $\mathrm{F}$ & $\mathrm{p}$ & $\mathrm{F}$ & $\mathrm{p}$ & & $\mathrm{F}$ & $P$ & \\
\hline TGMV & 0.143 & 0.71 & 0.074 & 0.93 & & 2.812 & 0.06 & \\
\hline TWMV & 0.083 & 0.77 & 0.042 & 0.96 & & 0.658 & 0.52 & \\
\hline SCGMV & 0.044 & 0.83 & 0.411 & 0.66 & & 1.105 & 0.33 & \\
\hline SCV & 0.239 & 0.63 & 0.651 & 0.52 & & 2.487 & 0.08 & \\
\hline Frontal & 0.228 & 0.63 & 0.326 & 0.72 & & 1.630 & 0.20 & \\
\hline Temporal & 0.095 & 0.76 & 1.603 & 0.20 & & 3.922 & 0.020 & $d$ \\
\hline Parietal & 0.025 & 0.88 & 0.211 & 0.81 & & 0.356 & 0.70 & \\
\hline Occipital & 2.964 & 0.09 & 5.044 & 0.007 & $a, b$ & 1.488 & 0.23 & \\
\hline Cingulate & 2.545 & 0.11 & 1.276 & 0.28 & & 6.213 & 0.002 & $c, d$ \\
\hline Hippocampus & 3.914 & 0.048 & 2.005 & 0.14 & & 3.968 & 0.019 & c \\
\hline Caudate & 0.307 & 0.58 & 0.277 & 0.76 & & 0.158 & 0.85 & \\
\hline Putamen & 0.266 & 0.61 & 0.309 & 0.74 & & 0.520 & 0.60 & \\
\hline Thalamus & 0.469 & 0.49 & 1.224 & 0.29 & & 0.727 & 0.48 & \\
\hline Amygdala & 3.045 & 0.08 & 1.601 & 0.20 & & 3.259 & 0.039 & \\
\hline
\end{tabular}

Abbreviations: TGMV = total gray matter volume; TWMV = total white matter volume; SCGMV = subcortical gray matter volume; $\mathrm{SCV}=$ sum cortical volume

a: Non-driver $<$ low-frequency driver $(p<0.05)$, b: Low-frequency driver $>$ high-frequency driver $(p<0.05)$, $c$ : Nondriver $<$ active driver $(p<0.05)$, d: Less-active driver $<$ active driver $(p<0.05)$. 

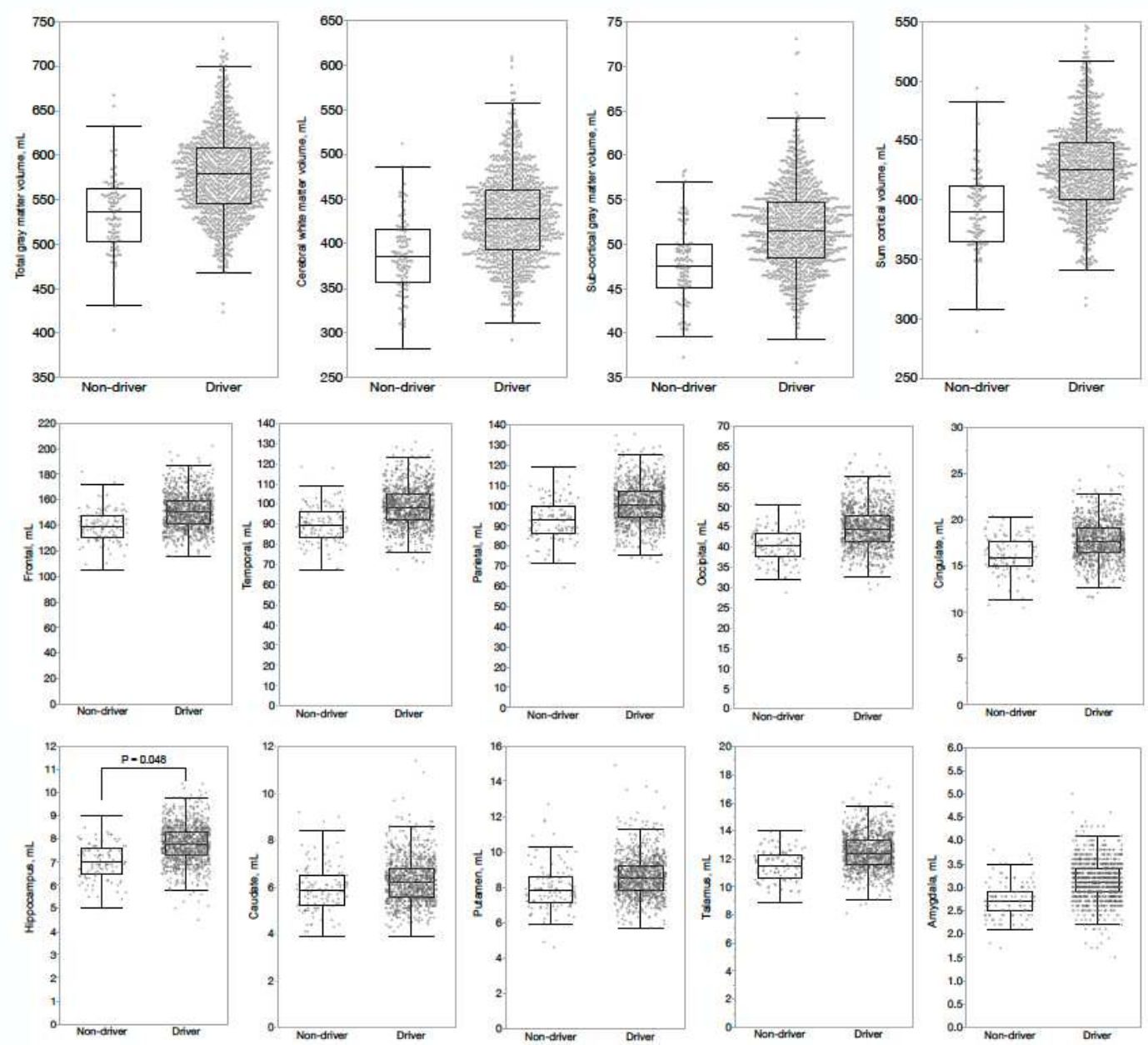

Figure 1. Comparison of regional brain volumes between drivers and non-drivers 
$\mathrm{R}$
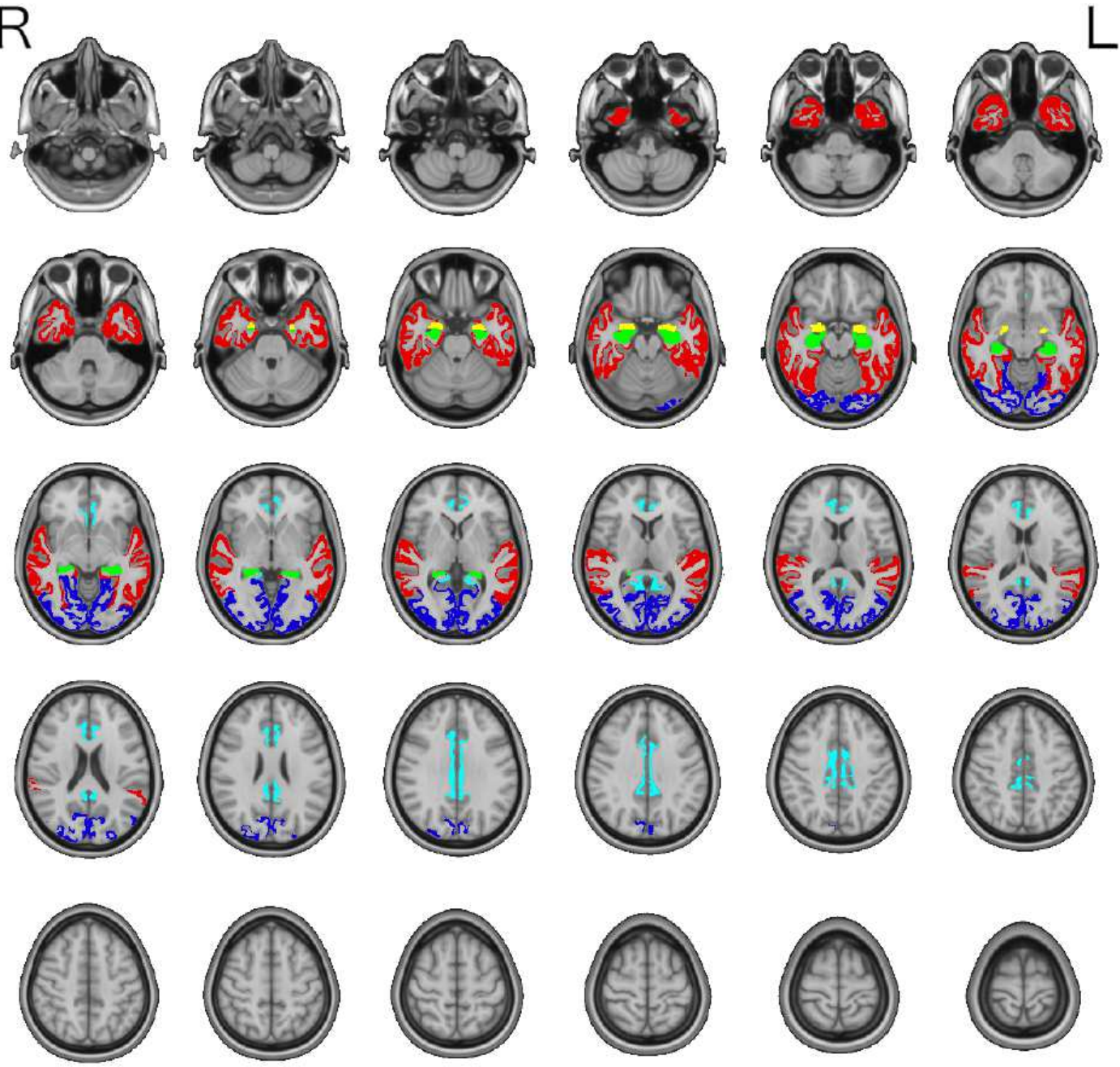

Figure 2. Brain regions with volume differences according to driving activity

Colors indicate the following ROIs: red = temporal cortex; blue = occipital cortex; light blue = cingulate cortex; green = hippocampus; yellow = amygdala. 